\title{
High-Order Sliding Mode Control for Networked Control System with Dynamic Noncooperative Game Scheduling
}

\author{
Weixuan Wang (D, Shousheng Xie, Bin Zhou, Jingbo Peng, Lei Wang, Hao Wang, \\ and Yu Zhang
}

Aeronautics Engineering College, Air Force Engineering University, Xi'an, China

Correspondence should be addressed to Weixuan Wang; wang_w_x@yeah.net

Received 15 December 2020; Revised 23 March 2021; Accepted 29 March 2021; Published 12 April 2021

Academic Editor: Xianming Zhang

Copyright (c) 2021 Weixuan Wang et al. This is an open access article distributed under the Creative Commons Attribution License, which permits unrestricted use, distribution, and reproduction in any medium, provided the original work is properly cited.

\begin{abstract}
Specific to the NCSs where sensor signals can be processed centrally, a collaborative design scheme of dynamic game scheduling and advanced control theory was proposed in the present study. Firstly, by using the Jordan standard state space equation of the research object, the three elements of state noncooperative game were built, and the existence and uniqueness of Nash equilibrium solution were verified. In addition, the iterative equation of the scheduling matrix was derived by complying with the designed utility function. Secondly, refer to the number of restricted states the order of sliding mode was determined. And based on it, the corresponding sliding surface was designed. Subsequently, the quadratic optimization theory was adopted to regulate the control value following the implementation of the scheduling strategy to ensure that the control quality was further enhanced in the limited network service. Lastly, a TrueTime simulation example is established to verify the effectiveness of the proposed scheme.
\end{abstract}

\section{Introduction}

Network control systems (NCSs), reflecting the development and integration of communication engineering, control science, and computer technology, have aroused considerable attention in numerous fields, such as manufacturing systems, process control, automobiles, automated highway systems, and unmanned aerial vehicles [1]. As opposed to the conventional point-to-point wiring, the NCSs integrate computer networks into control systems [2], thereby showing enormous advantages (e.g., lower cost, reduced weight and power, easier maintenance, remote control, higher reliability, and increased system flexibility [3]).

The insertion of communication networks, however, lands the control of NCSs into considerable difficulties. The primary challenge should be overcome is network-induced problem, including network-induced delays and packet dropouts. In addition, much effort has been devoted over the past few years (e.g., [4-13]).

The research enthusiasm of network-induced problems in NCSs reveals the contradiction between QoP (Quality of
Performance) and QoS (Quality of Service), i.e., the continuous improvement of QoP demand and the practical constraints of QoS guarantee [14]. To solve such a contradiction, researchers of this area focused on active network scheduling and collaborative control and achieved much progress. In [15], a sufficient condition was obtained to stabilize a continuous-time multi-input network system exhibiting stochastic uncertainties. It was suggested that the transmission scheduling and the controller should not be treated separately but jointly. In [16], the Round-Robin protocol was adopted to schedule the sensor transmissions, and the noncooperative optimal cooperative controller was designed in terms of a class of discrete time-varying NCSs under external disturbances. In [17], the event-triggered mechanism with the dynamic dwell-time switching rule was integrated and the design integrating scheduling and state feedback control for NCSs with time-delay was achieved.

Although the research of network scheduling and collaborative control has demonstrated an interesting potential, important fields still need to be further investigated to allow a wider practical deployment, especially in terms of the 
expansion of collaborative control methods and scheduling methods.

Sliding mode control (SMC), as a reliable and tried-andtrue method, was developed for the complex environment [18], and the cooperative controller design of NCSs with network scheduling can be theoretically supported. Reference [19] investigated the collaborative design of roundrobin protocol scheduling and static output-feedback SMC in terms of a type of uncertain control systems. The linear sliding mode surface and SMC law were designed by exploiting the measured output information and the actuator input, respectively; based on token-dependent Lyapunov functions the sufficient conditions for ensuring, the asymptotic stability of systems and the reachability of the sliding mode surface were determined. In [20], the stochastic communication protocol (SCP) was adopted to schedule data packets in the controller-actuator channel, and a tokendependent cooperative SMC law was formulated. Sufficient conditions were established for the reachability of specified sliding mode surface and the stochastic stability under a prescribed $\mathrm{H}$-infinity performance level.

Notably, SMC systems usually encounter a chattering phenomenon owing to the discontinuity of the control law, which can be regarded as the main drawback of the SMC. Therefore, various methodologies have been proposed to reduce the chattering phenomenon. In [21], the sign function was replaced by a sigmoid-like function to smooth the behaviors of the controller, which significantly reduce chattering. In [22] a novel SMC that employs extended disturbance observer was proposed to counteract the effect of uncertainties to enable a larger reduction in the magnitude of the discontinuous component in control, which can substantially alleviate the chattering problem. In addition, the research in [23] showed that compare to traditional SMC, the high-order sliding mode control (HOSMC) acts the discontinuous control variables on the high-order derivatives, so that the HOSMC not only preserves the main advantages of SMC but also improves the performance of the closed-loop system by reducing the chattering phenomenon and by increasing the accuracy. Therefore, it is significant to develop HOSMC as the method of collaborative controller.

The history of game theory can date back to von Neumann's 1928 paper [24]. This theory, one of the greatest theoretical achievements of the twentieth century, is currently in a branch of modern mathematics and has been developed by leaps and bounds.

As one of the most common branch of game theory, the noncooperative game refers to each participant acting independently, without collaboration or communication with any others, to maximize the use of game rules to maximize their own interests [25]. In other words, it focuses on the interests of individual participant and studies how participants make the most favorable decisions in the case of mutual influence or even competition.

At present, the noncooperative games research has progressively matured. By complying with the mastering degree on information of competitors, noncooperative games can fall to complete information and incomplete information game. Moreover, following the order of competitive action, it can fall to static and dynamic game. To be specific, complete information static game is widely applied to spectrum allocation, computer information, transmission through wireless network etc. In addition, incomplete information dynamic game is universal using in mechanical design, financial analysis etc.

Though it is applied in various fields, the basic issues concerned remain largely unchanged, i.e., numerous participants strive for several types of 'benefit' measures. However, given the limited resources, all competitors cannot be ensured to be able to get fair treatment [26]. Specific to the NCSs with network scheduling, as impacted by the limited bandwidth, network resource competition takes place among data packets, and several data packets may be discarded. Since there is no binding cooperation protocol among the competing packets, it is consistent between this kind of competition and the idea of noncooperative game. Accordingly, the idea of noncooperative game theory can be applied to determine whether the packets are sent. For instance, in [27], Tipsuwan considered the demand for equilibrium of bandwidth allocation in NCS. In accordance with game theory, a dynamic bandwidth allocation method was proposed to control bandwidths given to open-loop NCS at Nash equilibrium. In [28], Yan exploited the average sensitivities of reference in utility function to assess the effects exerted by network-induced delays. Moreover, by complying with the network pricing mechanism and game theory, the dynamic bandwidth resource allocation model of the overall control system for optimal performance was built.

However, all the mentioned researches on NCS game scheduling did not consider the cooperative design of the controller. Given this, the present study combined game theory to develop the collaborative design of non-cooperative dynamic game scheduling and SMC. Firstly, the research object was standardized, and the three elements of non-cooperative game were set. The Nash equilibrium equation and the scheduling iterative calculation equation were formulated, and then the existence and uniqueness of the Nash equilibrium solution were verified. Secondly, given the effect of the game scheduling and the control scheduling, the quadratic optimal time-varying sliding mode surface and the equivalent control adjustment item were created, and the research of HOSMC by complying with state game scheduling was completed. Lastly, a simulation example demonstrated the efficiency of the proposed method.

\section{Networked Control System Description}

In the present study, a specific model was developed with noncooperative dynamic game scheduling (Figure 1).

According to Figure 1, the output of the system was sent from plant to scheduler. After data interaction and short iterative calculations, the scheduler dynamically determined which state signal could be sent. At the other end of the network, the order of SMC was determined by the number of restricted states. Following the real-time situation of scheduling and quadratic optimal SMC theory, the control value was fine-tuned and whether it should be transmitted 


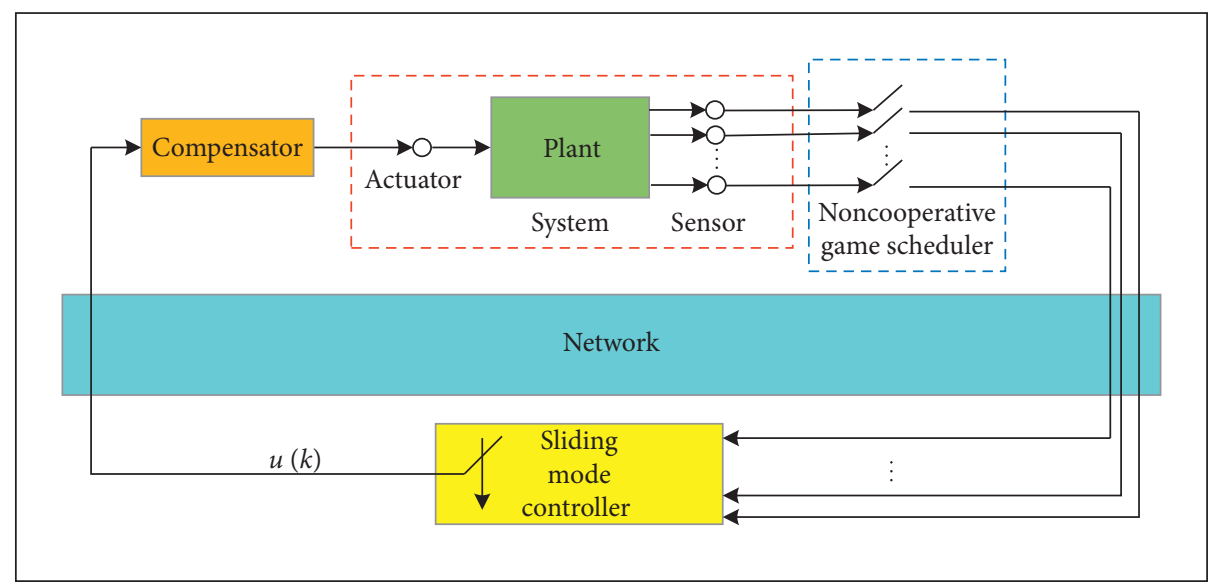

FIGURE 1: The structure of NCS with noncooperative game scheduling.

was judged. After returning to the bottom of the networked system, the switching items were compensated by compensator. The mentioned information is subsequently passed to actuator. Accordingly, a complete closed-loop network control technology route was achieved.

Based on the mentioned analysis, the discrete state-space equation could be determined, consisting of the corresponding modifications in controller input and output after scheduling restrictions. Since the present study is primarily concerned with the dynamic scheduling for state, the singleinput multiple-output system was taken as the research object. After defining the forward channel scheduling matrix $\Psi(k) \in\left\{\begin{array}{ll}0 & 1\end{array}\right\}^{m}$ composed of Booleans and the backward channel scheduling variable $M(k)=1-N(k) \in\left\{\begin{array}{ll}0 & 1\end{array}\right\}$, the NCS was determined, as described as follows:

$$
\left\{\begin{array}{l}
x(k+1)=A x(k)+B w(k) \\
u(k)=N(k) u(k-1)+M(k) w(k) \\
v(k)=\Psi(k) C^{\prime \prime} x(k)
\end{array}\right.
$$

where $x(k) \in R^{m}$ denotes state vector; $w(k), u(k), v(k) \in R$ represent the calculated control value, the transmission control value, and the actual input control value, respectively; $A, B$, and $C^{\prime \prime}$ denote constant matrices with appropriate dimensions.

Suppose system (1) is controllability. The nonsingular transformation $\tilde{x}(k)=\Upsilon x(k)$ was performed, and the Jordan standard form was obtained as

$$
\tilde{x}(k+1)=\widetilde{A}(k) \widetilde{x}(k)+\widetilde{B}(k) w(k),
$$

where $\quad \widetilde{A}(k)=\Upsilon A \Upsilon^{-1}=\left[\begin{array}{ccccc}0 & 1 & 0 & \ldots & 0 \\ 0 & 0 & 1 & \ldots & 0 \\ \vdots & \vdots & \vdots & \ddots & \vdots \\ -a_{1} & -a_{2} & -a_{3} & \ldots & -a_{m}\end{array}\right]$,

$$
\begin{aligned}
\widetilde{B}(k) & =\Upsilon B=\left[\begin{array}{c}
0 \\
0 \\
\vdots \\
1
\end{array}\right], \\
\Upsilon & =\left[\begin{array}{llll}
\gamma & \gamma A & \ldots & \gamma A^{m-1}
\end{array}\right]^{T}, \\
\gamma & =\left[\begin{array}{lllll}
0 & 0 & \ldots & 0 & 1
\end{array}\right]\left[\begin{array}{llllll}
B & A B & A^{2} B & \ldots & A^{m-1} B
\end{array}\right]^{-1} .
\end{aligned}
$$

\section{State Nonooperative Game Scheduling Design}

Denote $\tilde{x}=\left[\begin{array}{cc}\tilde{x}_{1}^{T} & \tilde{x}_{2}^{T}\end{array}\right]^{T}$, where $\widetilde{x}_{1} \in R^{m-1}, \widetilde{x}_{2} \in R$. Combined with the structure of $\widetilde{A}$ and $\widetilde{B}$, the system (2) can be decomposed into two subsystems:

$$
\begin{aligned}
& \tilde{x}_{1}(k+1)=\widetilde{A}_{11}(k) \tilde{x}_{1}(k)+\widetilde{A}_{12}(k) \tilde{x}_{2}(k), \\
& \tilde{x}_{2}(k+1)=\widetilde{A}_{21}(k) \widetilde{x}_{1}(k)+\widetilde{A}_{22}(k) \tilde{x}_{2}(k)+w(k),
\end{aligned}
$$

where (4a) and (4b) are the sliding mode dynamics and the reaching mode dynamics of system (2), respectively. $\widetilde{A}_{11}$, $\widetilde{A}_{12}, \widetilde{A}_{21}$, and $\widetilde{A}_{22}$ are submatrices of $\widetilde{A}$, such that $\widetilde{A}=\left[\begin{array}{cc}\widetilde{A}_{11} & \widetilde{A}_{12} \\ \widetilde{A}_{21} & \widetilde{A}_{22}\end{array}\right], \quad$ and $\quad \widetilde{A}_{11}=\left[\begin{array}{ccccc}0 & 1 & 0 & \ldots & 0 \\ 0 & 0 & 1 & \ldots & 0 \\ \vdots & \vdots & \vdots & \ddots & \vdots \\ 0 & 0 & 0 & \ldots & 1\end{array}\right] \epsilon$ $R^{(m-1) \times(m-1)}, \widetilde{A}_{12}=0 \in R^{m-1}, \widetilde{A}_{21}=\left[\begin{array}{llll}-a_{1} & -a_{2} & \ldots-a_{m-1}\end{array}\right]$, $\widetilde{A}_{22}=\left[-a_{m}\right]$.

To build the noncooperative game model, based on the equivalent control theory, the classic linear sliding surface is chosen:

$$
s(k)=\left[\begin{array}{ll}
C & I
\end{array}\right] \widetilde{x}(k)=C \tilde{x}_{1}(k)+\tilde{x}_{2}(k) .
$$

As the state trajectory of the system enters the sliding mode surface, we have $s(k+1)=s(k)=0$.

When $s(k)=0$,

$$
\tilde{x}_{2}(k)=-C \tilde{x}_{1}(k) .
$$

From (4a), (4b), and (5), it yields the following:

$$
\begin{aligned}
s(k+1)= & C\left(\widetilde{A}_{11}(k) \widetilde{x}_{1}(k)+\widetilde{A}_{12}(k) \widetilde{x}_{2}(k)\right)+\widetilde{A}_{21}(k) \widetilde{x}_{1}(k) \\
& +\widetilde{A}_{22}(k) \widetilde{x}_{2}(k)+w(k) .
\end{aligned}
$$

If all signals do not participate in the scheduling, as indicated by (2) and (7), there are parameters $\bar{a}_{i}(i=1,2, \ldots, m)$, so the equivalent control law in the sliding mode can be obtained as 


$$
u_{e q}(k)=\sum_{i=1}^{m} \bar{a}_{i} x_{i}(k)
$$

Based on the mentioned analysis, the following is the specific study on the noncooperative game for states.

In the NCS with $m$ sensor transmission nodes, the state sent by the $i_{\text {th }}$ node is expressed as $x_{i}(k)$, the contribution degree to the state gain of the plant at $k+1$ is denoted as $\kappa_{i}$. The network patency degree is defined as $\widehat{\Psi}_{i i}(k) \in[0,1]$. Subsequently, the state received by the controller is $\widehat{\Psi}_{i i}(k) x_{i}(k)$. Since $\bar{a}_{i}$ represents the correlation coefficient of the data of each sending node, the state contribution degree of other state information nodes to the controller is

$$
\sum_{j=1, j \neq i}^{m} \bar{a}_{j} \widehat{\Psi}_{j j}(k) x_{j}(k) .
$$

Subsequently, the contribution degree of the $i_{\text {th }}$ state node to the state gain of the controlled object is written as

$$
\kappa_{i}=\frac{\bar{a}_{i} \widehat{\Psi}_{i i}(k) x_{i}(k)}{\sum_{j=1, j \neq i}^{m} \bar{a}_{j} \widehat{\Psi}_{j j}(k) x_{j}(k)+\theta^{2}},
$$

where $\theta^{2}$ denotes the noise interference degree of the NCS.

The corresponding boundaries of the scheduling rules are defined as

$$
0 \leq \widehat{\Psi}_{i i}(k) \leq 1, \quad i \in\{1,2, \ldots, m\} .
$$

For the network scheduling in the present study, contribution degree of state gain corresponding to each state should have a certain threshold, as expressed as follows:

$$
\kappa_{i} \geq \kappa_{i}^{\text {th }}, \quad i \in\{1,2, \ldots, m\}
$$

where $\kappa_{i}^{\text {th }}$ refers to the threshold of contribution degree.

After clarifying the definition of contribution degree and related boundary conditions, the noncooperative game model of state scheduling is expressed as

$$
\Theta=\left[M,\left\{\widehat{\Psi}_{i i}\right\}_{i \in M},\left\{\varphi_{i}\right\}_{i \in M}\right],
$$

where the three elements of the game include

(1) Participant: $M \in\{1,2, \ldots, m\}$ denotes the set of all state variables participating in the game.

(2) Strategy space: $\widehat{\Psi}=\left[\widehat{\Psi}_{11}, \widehat{\Psi}_{22}, \ldots, \widehat{\Psi}_{m m}\right]$ represents the set of scheduling strategies provided by the respective state variable according to sliding mode equivalent control, where $\widehat{\Psi}_{i i}=[0,1]$ denotes the strategy space of the $i_{t h}$ state scheduling strategy.

(3) Utility function: $\varphi_{i}\left(\widehat{\Psi}_{i i}, \widehat{\Psi}_{-i i}\right)(\forall i \in M)$ refers to the utility function of each state scheduling variable. In such a state scheduling game, each scheduling variable attempts to maximize its respective utility function, as follows:

$$
\max _{\widehat{\Psi}_{i i} \in[0,1]} \varphi_{i}\left(\widehat{\Psi}_{i i}, \widehat{\Psi}_{-i i}\right), \quad \forall i \in M .
$$

Punishment critically impacts the healthy performance of noncooperative game scheduling. Figure 2 shows the relationship between utility function without penalty term and network patency degree. It can be seen that the higher the network patency degree is, the larger the utility function value is.

In this noncooperative game, each participant (i.e., state variable) will attempt to maximize its network patency degree (expressed as " 1 "), to improve their own interests (i.e., utility function value), and completely disregard the interests of others. Thus, improper competition can be easily caused, and then the final result cannot be obtained within finite iteration steps, demonstrating that the optimal global scheduling performance is difficult to ensure.

After a reasonable utility function penalty was set, the relationship between utility function and network patency degree is shown in Figure 3. It can be seen that there is an "opt" between " 0 " and " 1 ," which can maximize the value of the utility function.

In this noncooperative game, the network patency degree requirement of each state variable was reduced before "1." After this transformation, the state variable participation side, based on their actual contribution at the current moment, presented a matching "bid satisfaction point"-opt consciously, instead of uniformly requesting the maximum patency degree "1," thereby facilitating guidance positive competition to achieve a win-win situation for each other.

To enable the penalty factor to be adaptively adjusted, i.e., the control with a large contribution will be punished slightly, and the control with a small contribution will be punished slightly, utility function is designed as

$$
\begin{aligned}
\varphi_{i}\left(\widehat{\Psi}_{i i}, \widehat{\Psi}_{-i i}\right)= & \varepsilon_{i} \ln \left(\kappa_{i}-\kappa_{i}^{t h}\right)-\frac{\sigma_{i} \bar{a}_{i i} x_{i}(k)}{k} \\
& \cdot\left[\widehat{\Psi}_{i i}(k)+\sum_{j=1}^{k-1} \widehat{\Psi}_{i i}(j)-k \widehat{\Psi}_{\text {th }}\right] .
\end{aligned}
$$

where $\varepsilon_{i}$ denotes contribution degree regulatory factor; $\sum_{j=1}^{k-1} \widehat{\Psi}_{i i}(j)$ represents the sum of the $i_{\text {th }}$ scheduling value in history; $\widehat{\Psi}_{\text {th }}$ refers to the threshold of scheduling variable; and $\sigma_{i}$ denotes cost factor. When the control becomes stable, the contribution degree regulatory factor should adaptively increase to reduce system chattering.

Since the multioutput networked control game scheduling belongs to the Nash equilibrium of the combined strategy of multistate participation, any participant cannot unilaterally deviate from the Nash equilibrium and increase its own benefits, as expressed below:

$$
\varphi_{i}\left(\widehat{\Psi}_{i i}^{*}, \widehat{\Psi}_{-i i}^{*}\right) \geq \varphi_{i}\left(\widehat{\Psi}_{i i}, \widehat{\Psi}_{-i i}^{*}\right), \quad \forall \widehat{\Psi}_{i i},
$$

where $\widehat{\Psi}_{i i}^{*}$ represents the optimal scheduling strategy for the $i_{\text {th }}$ state given the scheduling strategies of others are $\widehat{\Psi}_{-i i}^{*}=\left(\widehat{\Psi}_{11}^{*}, \widehat{\Psi}_{22}^{*}, \ldots, \widehat{\Psi}_{(i-1)(i-1)}^{*}, \widehat{\Psi}_{(i+1)(i+1)}^{*}, \ldots, \widehat{\Psi}_{m m}^{*}\right)$.

For $\widehat{\Psi}_{i i}^{*}$ in (16), set the gradient of (15) to 0 ; it yields: 


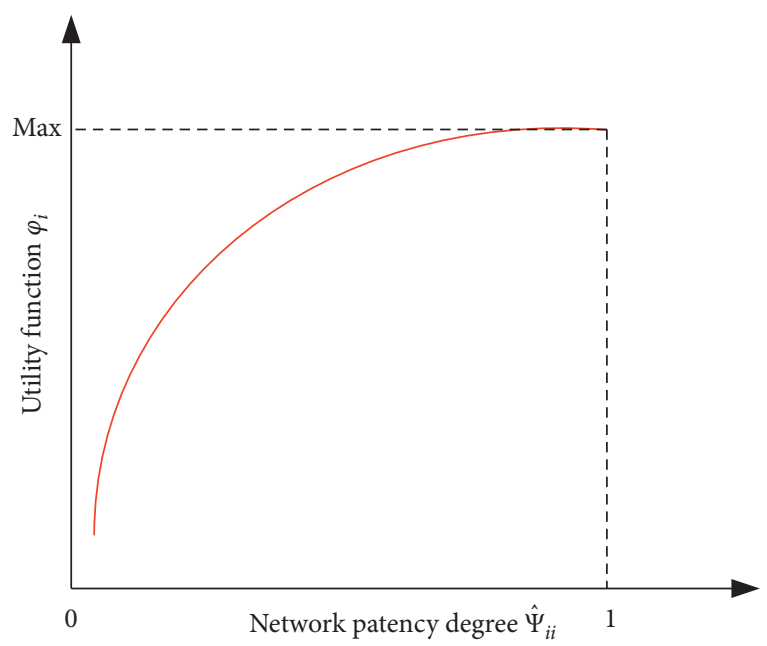

FIGURE 2: The relationship between utility function without penalty and network patency degree.

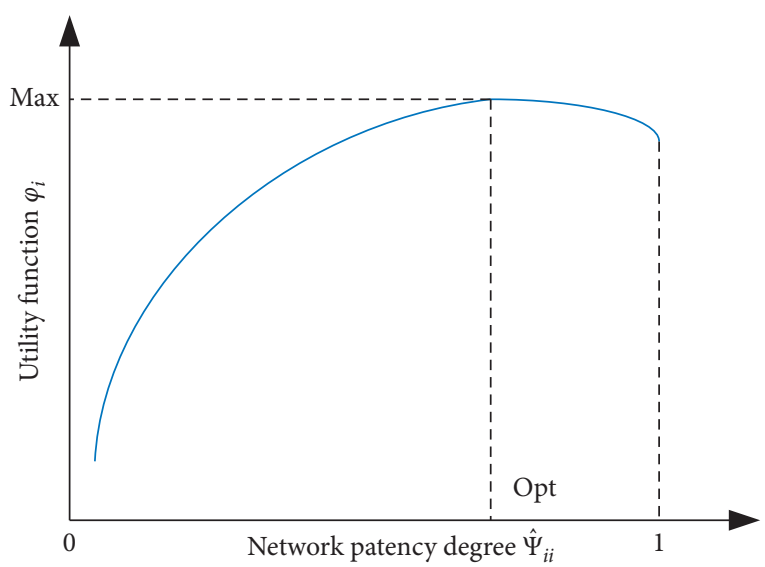

FIGURE 3: The relationship between utility function with penalty and network patency degree.

$$
\kappa_{i}=\frac{\kappa_{i}^{\text {th }}+\varepsilon_{i} k}{\sigma_{i}\left(\sum_{j=1, j \neq i}^{m} \bar{a}_{j} \widehat{\Psi}_{j j}(k) x_{j}(k)+\theta^{2}\right)} .
$$

Based on (10) and (17), the game iterative equation is yielded as

$$
L\left(\widehat{\Psi}_{i i}(k)\right)=\widehat{\Psi}_{i i}^{+}(k)=\frac{\varepsilon_{i} k}{\sigma_{i} \bar{a}_{i} x_{i}(k)}+\kappa_{i}^{\mathrm{th}} \frac{\widehat{\Psi}_{i i}(k)}{\kappa_{i}} .
$$

After determining the state scheduling regulation value $\phi(k)$ by complying with the network patency degree, the regularized state scheduling matrix iteration formula is yielded as

$$
\Psi_{i i}(k)=\left\{\begin{array}{l}
0, \quad \frac{\varepsilon_{i} k}{\sigma_{i} \bar{a}_{i i} x_{i}(k)}+\kappa_{i}^{\mathrm{th}} \frac{\widehat{\Psi}_{i i}(k)}{\kappa_{i}}<\phi(k), \\
1, \quad \frac{\varepsilon_{i} k}{\sigma_{i} \bar{a}_{i i} x_{i}(k)}+\kappa_{i}^{\mathrm{th}} \frac{\widehat{\Psi}_{i i}(k)}{\kappa_{i}} \geq \phi(k) .
\end{array}\right.
$$

The whole game process is illustrated in Figure 4. At each sampling time, the state signal was transmitted from the sensor side to the initialized noncooperative game scheduler. Subsequently, the iterative calculation of the scheduling matrix was initiated, and it was judged whether the sum of all contribution degree was maximized. Next, the network patency degree was transformed to the actual scheduling signal by (19). Lastly, the state variables participated in the competition were limited or sent to the controller based on their respective scheduling signals $(0 / 1)$, where 0 indicates that the state variable is limited and 1 indicates that the state variable is started to be sent.

Though the specific algorithm design and implementation steps of the state noncooperative game were completed as suggested above, the strategy should satisfy two prerequisites, i.e., the existence and uniqueness of the Nash equilibrium solution. The proof of both is elucidated below:

Theorem 1 (Existence). The equivalent conditions for the existence of a Nash equilibrium solution in the noncooperative game model of state network scheduling $\Theta=\left[N,\left\{\widehat{\Psi}_{i i}\right\}_{i \in M},\left\{\varphi_{i}\right\}_{i \in M}\right]$ are written as

(1) The scheduling matrix strategy space $\widehat{\Psi}_{i i}(k)$ is a nonempty bounded closed convex set on the Euclidean space.

(2) Utility function $\varphi_{i}\left(\widehat{\Psi}_{i i}, \widehat{\Psi}_{-i i}\right)$ is a continuous quasiconcave function on $\widehat{\Psi}_{i i}(k)$.

Proof. Since the strategy space of the respective state scheduling is on the interval $[0,1]$, condition (1) is verified.

The second-order derivative of the utility function is expressed as

$$
\frac{\partial \varphi_{i}^{2}}{\partial \widehat{\Psi}_{i i}^{2}}=-\frac{\varepsilon_{i}\left(\bar{a}_{i} x_{i}(k)\right)^{2}}{\left[\left(\sum_{j=1, j \neq i}^{m} \bar{a}_{j} \widehat{\Psi}_{j j}(k) x_{j}(k)+\theta^{2}\right)\left(\kappa_{i}-\kappa_{i}^{\text {th }}\right)\right]^{2}}<0 .
$$

Accordingly, the proof is completed.

Theorem 2. (Uniqueness [29]) Specific to noncooperative game scheduling, the Nash equilibrium algorithm in the present study converges to a unique point if and only if the following three conditions hold:

(1) Monotonicity, i.e., when $\widehat{\Psi}^{\prime}>\widehat{\Psi}$, one has $L\left(\widehat{\Psi}^{\prime}\right)>L(\widehat{\Psi})$.

(2) Positivity, i.e., $L(\widehat{\Psi})>0$.

(3) Scalability, i.e., $\forall \ell>1$, one has $\ell L(\widehat{\Psi})>L(\ell \widehat{\Psi})$.

Proof

(1) From the iterative equation (18), it yields 


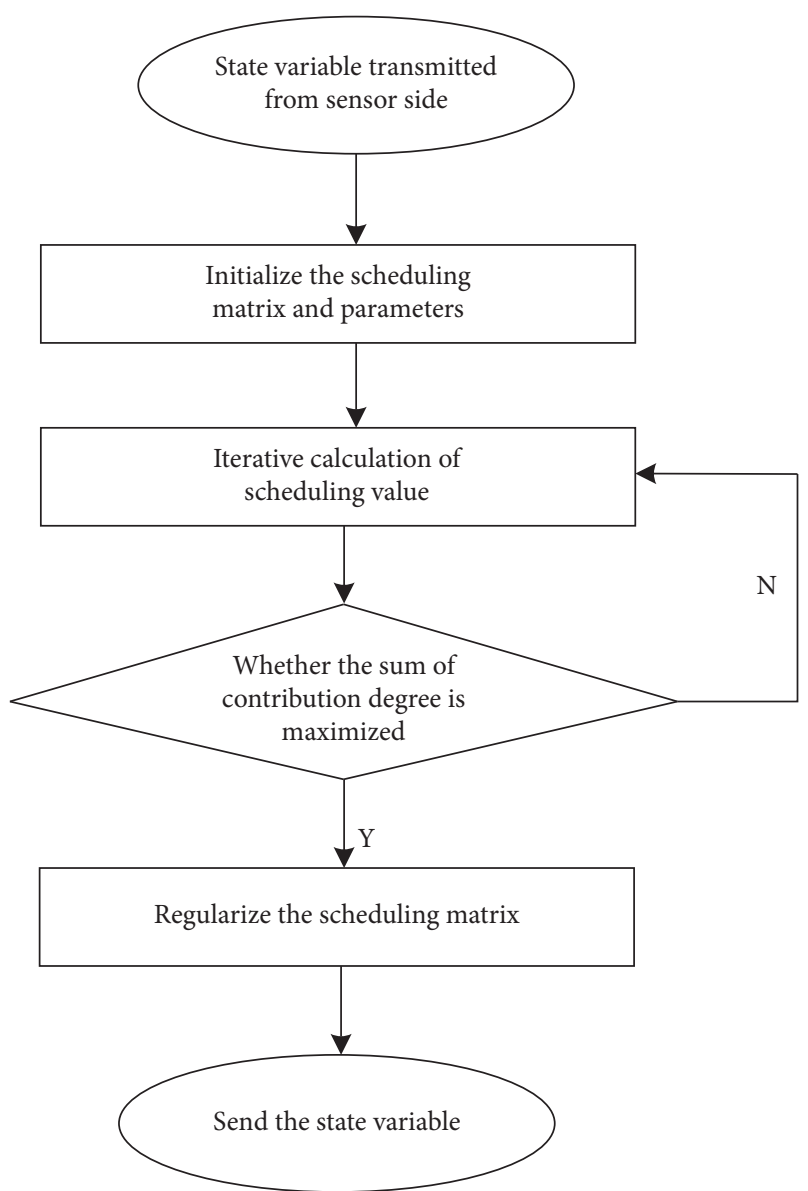

FIGURE 4: The implementation of noncooperative game scheduling for state.

$$
\begin{aligned}
L\left(\widehat{\Psi}^{\prime}\right)-L(\widehat{\Psi})= & \frac{\varepsilon_{i} k}{\sigma_{i} \bar{a}_{i} x_{i}(k)}+\kappa_{i}^{\mathrm{th}} \frac{\widehat{\Psi}_{i i}^{\prime}(k)}{\kappa_{i}} \\
& -\left[\frac{\varepsilon_{i} k}{\sigma_{i} \bar{a}_{i} x_{i}(k)}+\kappa_{i}^{\mathrm{th}} \frac{\widehat{\Psi}_{i i}(k)}{\kappa_{i}}\right]=\frac{\kappa_{i}^{\mathrm{th}}}{\kappa_{i}}\left(\widehat{\Psi}^{\prime}-\widehat{\Psi}\right) .
\end{aligned}
$$

Thus, when $\widehat{\Psi}^{\prime}>\widehat{\Psi}$, it yields that $L\left(\widehat{\Psi}^{\prime}\right)>L(\widehat{\Psi})$.

(2) From the definition of the parameters in the three elements of the noncooperative game in the present study, it yields

$$
L\left(\widehat{\Psi}_{i i}(k)\right)=\frac{\varepsilon_{i} k}{\sigma_{i} \bar{a}_{i} x_{i}(k)}+\kappa_{i}^{\text {th }} \frac{\widehat{\Psi}_{i i}(k)}{\kappa_{i}}>0 .
$$

(3) Since

$$
\begin{aligned}
\ell L(\widehat{\Psi})-L(\ell \widehat{\Psi})= & \ell\left[\frac{\varepsilon_{i} k}{\sigma_{i} \bar{a}_{i} x_{i}(k)}+\kappa_{i}^{t h} \frac{\widehat{\Psi}_{i i}(k)}{\kappa_{i}^{\Psi}}\right] \\
& -\left[\frac{\varepsilon_{i} k}{\sigma_{i} \bar{a}_{i} x_{i}(k)}+\kappa_{i}^{\text {th }} \frac{\ell \widehat{\Psi}_{i i}(k)}{\kappa_{i}^{\ell \Psi}}\right] \\
= & \frac{\varepsilon_{i} k}{\sigma_{i} \bar{a}_{i} x_{i}(k)}(\ell-1)+\ell \kappa_{i}^{\text {th }} \widehat{\Psi}\left(\frac{1}{\kappa_{i}^{\Psi}}-\frac{1}{\kappa_{i}^{\ell \widehat{\Psi}}}\right),
\end{aligned}
$$

$\forall \ell>1$, it yields that $\left(\varepsilon_{i} k / \sigma_{i} \bar{a}_{i} x_{i}(k)\right)(\ell-1)>0$.

Moreover, due to $\ell \widehat{\Psi}_{i i}>\widehat{\Psi}_{i i}$, then from (10), it yields that $\kappa_{i}^{\ell \Psi}>\kappa_{i}^{\Psi}$, i.e., $1 / \kappa_{i}^{\widehat{\Psi}}-1 / \kappa_{i}^{\ell \widehat{\Psi}}>0$, and then $\ell L(\widehat{\Psi})-L(\ell \widehat{\Psi})>0$. The proof is therefore completed.

\section{High-Order Sliding Mode Collaborative Controller Design}

Based on the linear sliding mode surface of equivalent control in (7), by complying with the definition of finite difference method [30], the corresponding order forward difference increment of the restricted state variables of $m-$ $\widehat{m}(\widehat{m} \leq m)$ in the current noncooperative game scheduling results was built as

$$
\Delta^{i} \widehat{\boldsymbol{s}}(k)=\sum_{i=1}^{m-\widehat{m}}(-1)^{i}\left(\begin{array}{c}
m-\widehat{m} \\
i
\end{array}\right) \widehat{\boldsymbol{s}}(k+i-m+\widehat{m}) .
$$

Subsequently, HOSMC coordinated with the scheduling strategy was defined.

Definition 1. (High-order sliding mode [31]) If both the initial linear sliding mode and its forward differential increment can satisfy the formula below:

$$
\left\{\begin{array}{l}
\widehat{s}(k)=\Delta \widehat{s}(k)=\Delta^{2} \widehat{s}(k)=\cdots \Delta^{m-\widehat{m}_{\widehat{s}}}(k)=0, \\
\Gamma(k)=\left\{x(k) \mid \sqrt{\sum_{i=0}^{m-\widehat{m}}\left[\Delta^{i} \widehat{s}(k)\right]^{2}} \leq \Theta\right\} .
\end{array}\right.
$$

It can be ensured that the system will not leave after entering the sliding mode motion, where $\Gamma(k)$ denotes the high-order sliding mode motion range and $\Theta$ represents the specific range parameter.

After clarifying the cooperative starting point of the noncooperative game scheduling strategy, the design of the specific controller is to be presented.

To calculate the sliding mode surface parameter $C$, the objective function of the optimization problem is defined as

$$
J=\sum_{k=1}^{\infty}\left[\tilde{x}_{1}^{T}(k) P \tilde{x}_{1}(k)+\tilde{x}_{2}^{T}(k) Q \tilde{x}_{2}(k)\right],
$$

where $P$ and $Q$ both denote the symmetric positive definite matrices with appropriate dimensions.

Theorem 3. Based on the optimization problem (26), the sliding mode surface parameter $C$ in the equivalent control law (6) of the systems (4a) and (4b) that integrated scheduling and control satisfies

$$
C=\left(Q+\widetilde{A}_{12}^{T} R \widetilde{A}_{12}\right)^{-1} \tilde{A}_{12}^{T} R \widetilde{A}_{11}
$$

where $R$ denotes a positive definite matrix, which is the solution to the Riccati equation: 


$$
R=\widetilde{A}_{11}^{T} R \widetilde{A}_{11}-\widetilde{A}_{11}^{T} R \widetilde{A}_{12}\left(Q+\widetilde{A}_{12}^{T} R \widetilde{A}_{12}\right)^{-1} \widetilde{A}_{12}^{T} R \widetilde{A}_{11}+P .
$$

Proof: According to the necessary conditions of optimal control, there exist functional boundary value conditions below:

$$
\left\{\begin{aligned}
\widetilde{x}_{1}(k+1) & =\widetilde{A}_{11} \widetilde{x}_{1}(k)-\widetilde{A}_{12} Q^{-1} \tilde{A}_{12}^{T} \lambda(k+1), \\
\lambda(k) & =P \widetilde{x}_{1}(k)+\widetilde{A}_{11}^{T} \lambda(k+1), \\
\tilde{x}_{1}(0) & =\widetilde{x}_{10}, \\
\lambda(\infty) & =0 .
\end{aligned}\right.
$$

Subsequently, the control law (5) can be transformed into

$$
\widetilde{x}_{2}(k)=-Q^{-1} \widetilde{A}_{12}^{T} \lambda(k+1) .
$$

Moreover, let $\lambda(k)=R \widetilde{x}_{1}(k)$, then

$$
\left\{\begin{aligned}
\tilde{x}_{2}(k) & =-\left(Q+\widetilde{A}_{12}^{T} R \widetilde{A}_{12}\right)^{-1} \widetilde{A}_{12}^{T} \widetilde{x}_{1}(k), \\
\lambda(k+1) & =R \widetilde{A}_{11}-R \widetilde{A}_{12} Q^{-1} \widetilde{A}_{12}^{T} \lambda(k+1) .
\end{aligned}\right.
$$

It yields

$$
\widetilde{A}_{12}^{T} \lambda(k+1)=\left(I+\widetilde{A}_{12}^{T} R \widetilde{A}_{12} Q^{-1}\right)^{-1} \widetilde{A}_{12} R \widetilde{A}_{11} \widetilde{x}_{1}(k) .
$$

Combining (29) and (32), it yields

$$
\begin{aligned}
R \widetilde{x}_{1}(k)= & P \widetilde{x}_{1}(k)+\widetilde{A}_{11}^{T} R \widetilde{x}_{1}(k+1)=P \widetilde{x}_{1}(k) \\
& +\widetilde{A}_{11}^{T} R \widetilde{A}_{11} \widetilde{x}_{1}(k)-\widetilde{A}_{11}^{T} R \widetilde{A}_{12} Q^{-1} \widetilde{A}_{12}^{T} \lambda(k+1) \\
= & P \widetilde{x}_{1}(k)+\widetilde{A}_{11}^{T} R \widetilde{A}_{11} \widetilde{x}_{1}(k)-\widetilde{A}_{11}^{T} R \widetilde{A}_{12} \\
& \left(Q+\widetilde{A}_{12}^{T} R \widetilde{A}_{12}\right) \widetilde{A}_{12} R \widetilde{A}_{11} \widetilde{x}_{1}(k) .
\end{aligned}
$$

From the formula above, equation (28) is obtained.

This completes the proof.

Since the state is restricted by game scheduling, only $\widehat{m}(\widehat{m} \leq m)$ state data packets arrive at the controller end, after setting the nontransmitted state value to zero, the sliding mode surface (5) is expressed as

$$
s(k)=c_{s 1} \widetilde{x}_{s 1}(k)+c_{s 2} \tilde{x}_{s 2}(k)+\cdots c_{s(\widehat{m}-1)} \widetilde{x}_{s(\widehat{m}-1)}(k)+\widetilde{x}_{2}(k) .
$$

Given the uncertainty of (34), the high-order sliding mode surface can be designed as

$$
\widehat{s}(k)=\widehat{C} \varsigma(k),
$$

where $\quad \widehat{C}=\left[\begin{array}{lllll}\widehat{c}_{s 1} & \widehat{c}_{s 2} & \ldots & \widehat{c}_{s(m-\widehat{m})} & 1\end{array}\right], \quad \varsigma(k)=\left[\begin{array}{lll}s_{1}(k) & \end{array}\right.$ $\left.s_{2}(k) \cdot s_{m-\hat{m}+1}(k)\right]^{T}, \quad$ AND $\quad s_{i}(k)=s(k+i-m+\widehat{m}-1)$, $(i=1, \ldots, m-\widehat{m}+1)$.

According to the result of the dynamic game scheduling above, the state vector $\tilde{x}(k)$ can fall to two parts: $z(k)=\left[\begin{array}{lllll}\tilde{x}_{\mathrm{s} 1}(k) & \tilde{x}_{\mathrm{s} 2}(k) & \ldots & \tilde{x}_{s(\hat{m}-1)}(k)\end{array}\right]^{T}$ and $z^{\prime}(k)=\left[\begin{array}{lll}\tilde{x}_{\mathrm{s} m} & \end{array}\right.$ $\left.(k) \widetilde{x}_{s(\hat{m}+1)}(k) \cdot \tilde{x}_{s m}(k)\right]^{T}$. In addition, define the augmented state vector $\bar{z}(k)=\left[\begin{array}{ll}z^{T}(k) & \varsigma^{T}(k)\end{array}\right]^{T}$, which satisfies

$$
\bar{z}(k)=\Omega\left[\begin{array}{c}
z(k) \\
z^{\prime}(k)
\end{array}\right],
$$

where $\Omega=\left[\begin{array}{cc}I_{\widehat{m}-1} & 0 \\ \Omega_{1} & \Omega_{2}\end{array}\right]$,

$$
\Omega_{1}=\left[\begin{array}{ccccc}
c_{s 1} & c_{s 2} & c_{s 3} & \ldots & c_{s(\widehat{m}-1)} \\
0 & c_{s 1} & c_{s 2} & \ldots & c_{s(\widehat{m}-2)} \\
0 & 0 & c_{s 1} & \ldots & c_{s(\widehat{m}-3)} \\
\vdots & \vdots & \vdots & \ddots & \vdots \\
0 & 0 & 0 & \ldots & \ldots
\end{array}\right] \text {, }
$$

$$
\Omega_{2}=\left[\begin{array}{ccccc}
1 & 0 & 0 & \ldots & c_{s(\widehat{m}-1)} \\
c_{s(\widehat{m}-1)} & 1 & 0 & \ldots & c_{s(\widehat{m}-2)} \\
c_{s(\widehat{m}-2)} & c_{s(\widehat{m}-1)} & 1 & \ldots & c_{s(\widehat{m}-3)} \\
\vdots & \vdots & \vdots & \ddots & \vdots \\
c_{s 2} & c_{s 3} & c_{s 4} & \ldots & 1
\end{array}\right] .
$$

Subsequently, system (2) can be transformed into

$$
\bar{z}(k+1)=\widehat{A}(k) \bar{z}(k)+\widehat{B}(k) w(k),
$$

where $\widehat{A}(k)=\Omega \widetilde{A}(k) \Omega^{-1}$ and $\widehat{B}(k)=\Omega \widetilde{B}(k)$.

Likewise, decompose the augmented state equation (37) as follows:

$$
\begin{aligned}
& z(k+1)=\widehat{A}_{11}(k) z(k)+\widehat{A}_{12}(k) \varsigma(k), \\
& \varsigma(k+1)=\widehat{A}_{21}(k) z(k)+\widehat{A}_{22}(k) \varsigma(k)+\widehat{B}(k) w(k),
\end{aligned}
$$

where $\widehat{B}=\left[\begin{array}{llll}0 & \ldots & 0 & 1\end{array}\right]^{T}$, 


$$
\begin{aligned}
& \widehat{A}_{11}=\left[\begin{array}{cccccc}
0 & 1 & 0 & \ldots & 0 & 0 \\
0 & 0 & 1 & \ldots & 0 & 0 \\
\vdots & \vdots & \vdots & \ddots & \vdots & \vdots \\
0 & 0 & 0 & \ldots & 0 & 1 \\
-c_{s 1} & -c_{s 2} & -c_{s 3} & \ldots & -c_{s(\widehat{m}-2)} & -c_{s(\widehat{m}-1)}
\end{array}\right], \\
& \widehat{A}_{12}=\left[\begin{array}{ccccc}
0 & 0 & \ldots & 0 & 0 \\
0 & 0 & \ldots & 0 & 0 \\
\vdots & \vdots & \ddots & \vdots & \vdots \\
0 & 0 & \ldots & 0 & 0 \\
1 & 0 & \ldots & 0 & 0
\end{array}\right] \\
& \widehat{A}_{21}=\left[\begin{array}{cccc}
0 & 0 & \ldots & 0 \\
0 & 0 & \ldots & 0 \\
\vdots & \vdots & \ddots & \vdots \\
0 & 0 & \ldots & 0 \\
-\widehat{a}_{1} & -\widehat{a}_{2} & \ldots & -\widehat{a}_{\widehat{m}-1}
\end{array}\right] \text {, } \\
& \widehat{A}_{22}=\left[\begin{array}{cccccc}
0 & 1 & 0 & \ldots & 0 & 0 \\
0 & 0 & 1 & \ldots & 0 & 0 \\
\vdots & \vdots & \vdots & \ddots & \vdots & \vdots \\
0 & 0 & 0 & \ldots & 0 & 1 \\
-\widehat{a}_{\hat{m}} & -\widehat{a}_{\hat{m}+1} & -\widehat{a}_{\widehat{m}+2} & \ldots & -\widehat{a}_{m-1} & -\widehat{a}_{m}
\end{array}\right] \text {. }
\end{aligned}
$$

In brief, with the transmission of state restricted by game scheduling, the high-order sliding mode method is suitable for timely adjustment of its impact on system control performance.

Since only part of the state value reaches the controller end after network scheduling, the equivalent control for (40) is designed as

$$
u_{e q}(k)=u_{\widehat{m}}(k)+u_{\Psi}(k),
$$

where $u_{\widehat{m}}(k)$ denotes the state feedback control value transmitted to the controller end and $u_{\Psi}(k)$ represents an adjustment item determined according to the result of game scheduling for control.

Accordingly, the augmented system (40) corresponding to the equivalent control law (42) can fall to

$$
\begin{aligned}
0 & =\widehat{A}_{21}(k) z(k)+\widehat{B}(k) u_{\widehat{m}}(k), \\
\varsigma(k+1) & =\widehat{A}_{22}(k) \varsigma(k)+\widehat{B}(k) u_{\Psi}(k) .
\end{aligned}
$$

This is different from (8). According to the form of the coefficient matrix, (43) can be solved as

$$
u_{\widehat{m}}(k)=\sum_{i=1}^{\widehat{m}-1} \widehat{a}_{i} x_{i}(k) \text {. }
$$

For the coordinated control adjustment item, emphatically analysis should be conducted. For easier derivation, (44) is decomposed again as follows:

$$
\begin{aligned}
& \varsigma_{1}(k+1)=\Lambda_{11}(k) \varsigma_{1}(k)+\Lambda_{12}(k) \varsigma_{2}(k), \\
& \varsigma_{2}(k+1)=\Lambda_{21}(k) \varsigma_{1}(k)+\Lambda_{22}(k) \varsigma_{2}(k)+u_{\Psi}(k),
\end{aligned}
$$

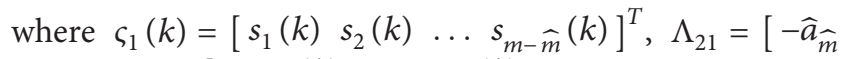
$\left.-\widehat{a}_{\hat{m}+\mathrm{r}}+-\widehat{a}_{m-1}\right], \quad \varsigma_{2}(k)=s_{m-\widehat{m}+1}(k), \quad \Lambda_{22}=-\widehat{a}_{m}, \quad \Lambda_{12}=$ $\left[\begin{array}{llll}0 & 0 & \ldots & 1\end{array}\right]^{T} \in R^{(m-\widehat{m}+1) \times 1}, \quad$ and $\quad \Lambda_{11}=$ $\left[\begin{array}{ccccc}0 & 1 & 0 & \ldots & 0 \\ 0 & 0 & 1 & \ldots & 0 \\ \vdots & \vdots & \vdots & \ddots & \vdots \\ 0 & 0 & 0 & \ldots & 1\end{array}\right] \in R^{(m-\widehat{m}) \times(m-\widehat{m})}$.

Based on the decomposition above, the high-order sliding surface (35) is expressed as

$$
\widehat{s}(k)=\left[\begin{array}{ll}
\widetilde{C} & I
\end{array}\right]\left[\begin{array}{lll}
\varsigma_{1}(k) & \varsigma_{2}(k)
\end{array}\right]^{T},
$$

where $\widetilde{C}=\left[\begin{array}{llll}\widehat{c}_{s 1} & \widehat{c}_{s 2} & \ldots & \widehat{c}_{s(m-\widehat{m})}\end{array}\right]$.

Since the control network scheduling was considered here as well, the designed scheduling value $M(k)$ could determine whether the control signal is maintained by the $\mathrm{ZOH}$ at the other end of the bus. Thus, the control value in (39) is equated with

$$
u_{\hat{m}}(k)+u_{\Psi}(k)=u_{M=1}(k)+u_{M=0}(k)=u_{M=1}(k)+u_{M=0}(k-1) \text {. }
$$

Based on (48), the state scheduling adjustment item is yielded as

$$
u_{\Psi}(k)=u_{M=1}(k)-u_{\widehat{m}}(k)+u_{M=0}(k-1) .
$$

Optimal SMC problem with this rule will lastly involve the association between $\varsigma(k)$ and $u_{M=1}(k)$, so the optimization goal (51) obtains the minimum value under the constraint (44).

According to (26), the optimal control target (51) is designed to ensure the asymptotic stability of the systems (46) and (47) and to ensure the state trajectory can reach the high-order sliding mode surface $\widehat{s}(\widehat{k}+1)=0$ in finite time $\widehat{k}$.

$$
J=\sum_{k=1}^{\widehat{k}}\left[\varsigma^{T}(k) P \varsigma(k)+u_{M=1}^{T}(k) Q u_{M=1}(k)\right] .
$$

Theorem 4. In finite number $\mathfrak{\Im}$ of iterations, the timevarying optimal high-order sliding mode switching surface for system (44) that considers control scheduling and satisfies the minimum quadratic optimization goal (51) is expressed as

$$
\begin{aligned}
\left(\widetilde{C} \Lambda_{12} \widetilde{C}+\Lambda_{22} \widetilde{C}-\widetilde{C} \Lambda_{11}-\Lambda_{21}\right) \varsigma_{1}(k)+W^{-1} \widehat{B}^{T} H \\
\cdot\left[\widehat{A}_{22} \varsigma(k)+\widehat{B}\left(u_{M=0}(k-1)-u_{\widehat{m}}(k)\right)\right] \\
+W^{-1} \widehat{B}^{T} f^{(\Im)}(k+1)+u_{\widehat{m}}(k)-u_{M=0}(k-1)=0,
\end{aligned}
$$

where 


$$
\begin{aligned}
f^{(i)}(k) & =\widehat{A}_{22}^{T} D\left[H \widehat{B}\left(u_{M=0}(k-1)-u_{\widehat{m}}(k)\right)+f^{(i)}(k+1)\right], \\
f^{(i)}(\infty) & =0, \quad i=1,2, \ldots,
\end{aligned}
$$

where $D=I-H \widehat{B} W^{-1} \widehat{B}^{T}, W=Q+\widehat{B}^{T} H \widehat{B}$, and $H$ denotes the unique positive definite solution of the following Riccati equation:

$$
\widehat{A}_{22}^{T} H \widehat{A}_{22}-H-\widehat{A}_{22}^{T} H \widehat{B} W^{-1} \widehat{B}^{T} H \widehat{A}_{22}+P=0,
$$

$\varsigma^{(i)}(k)$ represents the solution of following difference equation:

$$
\begin{aligned}
\varsigma^{(i)}(k+1)= & \left(I-\widehat{B} W^{-1} \widehat{B}^{T} H\right) \\
& \cdot\left[\widehat{A}_{22} \varsigma^{(i)}(k)+\widehat{B}\left(u_{M=0}(k-1)-u_{\widehat{m}}(k)\right)\right] \\
& -\widehat{B} W^{-1} \widehat{B}^{T} f^{(i)}(k+1) .
\end{aligned}
$$

Moreover, $u_{\Psi}(k)=\left(\widetilde{C} \Lambda_{12} \widetilde{C}+\Lambda_{22} \widetilde{C}-\widetilde{C} \Lambda_{11}-\Lambda_{21}\right) \varsigma_{1}(k)$ denotes the corresponding state scheduling adjustment item.
Proof. According to the necessary conditions of optimal control, there are functional boundary value conditions as follows:

$$
\left\{\begin{array}{l}
\varsigma(k+1)=\widehat{A}_{22}(k) \varsigma(k)+\widehat{B}(k)\left[u_{M=0}(k-1)-u_{\widehat{m}}(k)\right] \\
-\widehat{B}(k) Q^{-1} \widehat{B}^{T}(k) \lambda(k+1), \\
\lambda(k)=P \varsigma(k)+\widehat{A}_{22}^{T}(k) \lambda(k+1), \\
\varsigma(0)=\varsigma_{0}, \\
\lambda(\infty)=0 .
\end{array}\right.
$$

After $i$ iterations, the optimal high-order sliding mode switching surface satisfies

$$
Q u_{M=1}^{(i)}(k)+\widehat{B}^{T}(k) \lambda^{(i)}(k+1)=0 .
$$

Thus,

$$
\lambda^{(i)}(k)=H \varsigma^{(i)}(k)+f^{(i)}(k),
$$

where $H$ and $f^{(i)}(k)$, respectively, denote symmetric matrix and the $i_{\text {th }}$ iteration adjoint vector to be designed.

By combining (58) and (56), it yields

$$
\begin{aligned}
& \widehat{B}^{T} \lambda^{(i)}(k+1)=\left(I+\widehat{B}^{T} H \widehat{B} Q^{-1}\right)^{-1} \widehat{B}^{T}\left[H \widehat{A}_{22} \varsigma^{(i)}(k)+H \widehat{B}\left(u_{M=0}(k-1)-u_{\widehat{m}}(k)\right)+f^{(i)}(k+1)\right] \\
& \widehat{A}_{22}^{T} D\left[H \widehat{B}\left(u_{M=0}(k-1)-u_{\widehat{m}}(k)\right)+f^{(i)}(k+1)\right]-\left(H-P-\widehat{A}_{22}^{T} D H \widehat{A}_{22}\right) \varsigma^{(i)}(k)-f^{(i)}(k) \equiv 0 .
\end{aligned}
$$

Then, (53) and (54) hold.

In addition, $H$ is the unique positive definite solution of the Riccati equation. From (58) and $\widehat{B}^{(0)}\left(u_{M=0}^{(0)}-\right.$ $\left.u_{\stackrel{m}{(0)}}(k)\right)=0$, it yields that $f^{(0)}(k)=0$. Since the control value at the previous moment $u_{M=0}(k-1)$ and the state feedback control value passed through the scheduling $u_{\widehat{m}}(k)$ are known, the $i_{\text {th }}$ linear nonhomogeneous adjoint vector difference equation (53) can be iteratively yielded.

By combining (56), (57), and (59), the difference equation (55) holds.

According to $\varsigma^{(i)}(k)$, the optimal sliding mode switching surface is yielded as

$$
\begin{aligned}
& W u_{M=1}^{(i)}(k)+\widehat{B}^{T} H\left[\widehat{A}_{22} \varsigma^{(i)}(k)+\widehat{B}\left(u_{M=0}(k-1)-u_{\widehat{m}}(k)\right)\right] \\
& \quad+\widehat{B}^{T} f^{(i)}(k+1)=0 .
\end{aligned}
$$

After a certain number of iterations, the following final sliding mode switching surface is obtained:

$$
\begin{aligned}
& W u_{M=1}(k)+\widehat{B}^{T} H\left[\widehat{A}_{22} \varsigma(k)+\widehat{B}\left(u_{M=0}(k-1)-u_{\hat{m}}(k)\right)\right] \\
& +\widehat{B}^{T} \lim _{i \longrightarrow \infty} f^{(i)}(k+1)=0 .
\end{aligned}
$$

By complying with $(47), \varsigma_{2}(k)=-\widetilde{C} \varsigma_{1}(k)$ is yielded. To ensure $\varsigma(k)$ is always on the high-order sliding mode surface, $\widehat{s}(k+1)=0$. And, the corresponding equivalent control is

$$
\bar{u}_{e q}(k)=\left(\widetilde{C} \Lambda_{12} \widetilde{C}+\Lambda_{22} \widetilde{C}-\widetilde{C} \Lambda_{11}-\Lambda_{21}\right) \varsigma_{1}(k) .
$$

Given the optimal HOSMC theory, there is $\bar{u}_{e q}(k)=u_{\Psi}(k)$. And, because infinite iteration is unlikely to achieve, $\lim _{i \rightarrow \infty} f^{(i)}(k+1)$ is replaced by the calculation result $f^{(\mathfrak{J})}(k+1)$ of the iteration number $\mathfrak{\Im}$ under the error allowance. Thus, (62) is written as

$$
\begin{aligned}
- & W^{-1} \widehat{B}^{T} H\left[\widehat{A}_{22} \varsigma(k)+\widehat{B}\left(u_{M=0}(k-1)-u_{\widehat{m}}(k)\right)\right] \\
& -W^{-1} \widehat{B}^{T} f^{(\widetilde{\Im})}(k+1)-u_{\widehat{m}}(k)+u_{M=0}(k-1) \\
= & \left(\widetilde{C} \Lambda_{12} \widetilde{C}+\Lambda_{22} \widetilde{C}-\widetilde{C} \Lambda_{11}-\Lambda_{21}\right) \varsigma_{1}(k) .
\end{aligned}
$$

Subsequently, the time-varying optimal high-order sliding mode switching surface (52) can be obtained easily.

This completes the proof.

Since the control value is limited by network scheduling, after the mentioned optimal high-order sliding mode equivalent control design, the sliding mode switching control component is incorporated into the compensator on the actuator side: 


$$
u(k)=u_{e q}(k)+u_{s w}(k)
$$

where $u_{s w}(k)$ denotes the designed sliding mode switching control component as

$$
u_{s w}(k)=-\beta \operatorname{sgn} \widehat{s}(k) .
$$

Since the switching component does not affect the form of control, according to the decomposition system (44), it yields

$$
\varsigma(k+1)=\widehat{A}_{22} \varsigma(k)+\widehat{B} u_{\Psi}(k)-\beta \widehat{B} \operatorname{sgn} \widehat{s}(k) .
$$

Lemma 1. [32] For a dynamic system as $s^{\prime}(k+$ $1)=s^{\prime}(k)+g^{\prime}(k)-\beta^{\prime}$ sgns $s^{\prime}(k)$, if there are positive constants $a^{\prime}$ and $b^{\prime}$, such that $\left|g^{\prime}(k)\right|<a^{\prime}<b^{\prime}$, then $s^{\prime}(k)$ will converge to $\left|s^{\prime}(k)\right| \leq a^{\prime}+b^{\prime}<2 b^{\prime}$.

Lastly, according to Lemma 1, the design theorem for the compensation component of the switching term under the noncooperative game scheduling in the present study can be proposed in the following:

Theorem 5. For the system (44) that considers control scheduling, the equivalent control law is formulated according to Theorem 4, and the switching control compensation item (66) is adopted on the actuator side. If the sliding mode switching parameters satisfy,

$$
\beta>\operatorname{Sup}\left\|\left(\widehat{C} \widehat{A}_{22}-\widehat{C}\right) \varsigma(k)+\widehat{C} \widehat{B} u_{\Psi}(k)\right\|,
$$

$\varsigma(k), z(k)$, and $z^{\prime}(k)$, the state of augmented systems (38), (39), and (40) will have the stable boundaries as follows:

$$
\begin{aligned}
\|\varsigma(k)\| & \leq \frac{(\alpha+\beta)}{\left(1-\left\|\widehat{A}_{22}\right\|\right)}, \\
\|z(k)\| & \leq \frac{(\alpha+\beta)\left\|\widehat{A}_{12}\right\|}{\left[\left(1-\left\|\widehat{A}_{11}\right\|\right)\left(1-\left\|\widehat{A}_{22}\right\|\right)\right]}, \\
\left\|z^{\prime}(k)\right\| & \leq \frac{(\alpha+\beta)\left\|\Omega_{2}^{-1} \Omega_{1}\right\|\left\|\widehat{A}_{12}\right\|}{\left[\left(1-\left\|\widehat{A}_{11}\right\|\right)\left(1-\left\|\widehat{A}_{22}\right\|\right)\right]+\left((\alpha+\beta)\left\|\Omega_{2}^{-1}\right\| /\left(1-\left\|\widehat{A}_{22}\right\|\right)\right)} .
\end{aligned}
$$

Moreover, there is the optimal high-order sliding mode chattering boundary $|\widehat{s}(k)| \leq 2 \beta$.

Proof. Take the norm on both sides of (44) and it yields

$$
\|\varsigma(k+1)\| \leq\left\|\widehat{A}_{22}\right\|\|\varsigma(k)\|+\|\widehat{B}\|\left\|u_{\Psi}(k)\right\|+\beta\|\widehat{B}\|\|\operatorname{sgn} \widehat{s}(k)\| .
$$

Since $\|\operatorname{sgn} \widehat{s}(k)\|=1,\|\widehat{B}\|=1$, it yields

$$
\left\|_{\varsigma}(k+1)\right\| \leq\left\|\widehat{A}_{22}\right\|\left\|_{\varsigma}(k)\right\|+\left\|u_{\Psi}(k)\right\|+\beta .
$$

After determining the scheduling matrix $\Psi(k)$ according to game theory and determining the specific norm value $\alpha$ of the control variable scheduling adjustment item $u_{\Psi}(k)$ in accordance with the optimal HOSMC theory, the following inequality relationship holds:

$$
\begin{aligned}
\|\varsigma(\eta)\| \leq & \left\|\widehat{A}_{22}\right\|^{\eta}\|\varsigma(0)\| \\
& +\sum_{i=1}^{\eta-1}\left\|\widehat{A}_{22}\right\|^{i}(\alpha+\beta) \leq\left\|\widehat{A}_{22}\right\|^{\eta}\|\varsigma(0)\|+(\alpha+\beta) \frac{1-\left\|\widehat{A}_{22}\right\|^{\eta}}{1-\left\|\widehat{A}_{22}\right\|} .
\end{aligned}
$$

Likewise, from (39) it yields

$$
\|z(\eta)\| \leq\left\|\widehat{A}_{11}\right\|^{\eta}\|z(0)\|+\left\|\widehat{A}_{12}\right\|\|\varsigma(k)\| \frac{1-\left\|\widehat{A}_{11}\right\|^{\eta}}{1-\left\|\widehat{A}_{11}\right\|} .
$$

Designing $A$ and $C$, so that the spectral radius of $\widehat{A}_{22}$ and $\widehat{A}_{11}$ is less than 1 , it yields

$$
\begin{gathered}
\|\varsigma(k)\| \leq \frac{\alpha+\beta}{1-\left\|\widehat{A}_{22}\right\|}, \\
\|z(k)\| \leq \frac{\left\|\widehat{A}_{12}\right\|\|\varsigma(k)\|}{1-\left\|\widehat{A}_{11}\right\|} .
\end{gathered}
$$

According to the conversion relationship (36),

$$
z^{\prime}(k)=-\Omega_{2}^{-1} \Omega_{1} z(k)+\Omega_{2}^{-1} \varsigma(k)
$$

Next,

$$
\left\|z^{\prime}(k)\right\| \leq\left\|\Omega_{2}^{-1} \Omega_{1}\right\|\|z(k)\|+\left\|\Omega_{2}^{-1}\right\|\|\varsigma(k)\| .
$$

When the optimal high-order sliding mode motion is asymptotically stable, it yields that $\widehat{s}(k+1)-\widehat{s}(k)<0$. From (35) and (67),

$$
\begin{aligned}
\widehat{s}(k+1)= & \widehat{s}(k)+\widehat{C} \widehat{A}_{22} \varsigma(k)+\widehat{C} \widehat{B} u_{\Psi}(k)-\beta \widehat{C} \widehat{B} \operatorname{sgn} \widehat{s}(k), \\
& \cdot\left(\widehat{C} \widehat{A}_{22}-\widehat{C}\right) \varsigma(k)+\widehat{C} \widehat{B} u_{\Psi}(k)<\beta \widehat{C} \widehat{B} \operatorname{sgn} \widehat{s}(k) .
\end{aligned}
$$

As impacted by $\|\widehat{C} \widehat{B}\|=1$, (68) can be yielded by taking the norm on the both sides of the mentioned formula.

In addition, with Lemma 1 , it yields that $|\widehat{s}(k)| \leq 2 \beta$.

The proof is therefore completed.

\section{Simulation and Result Analysis}

To verify the effectiveness of the proposed noncooperative game scheduling and HOSMC strategy, a TrueTime simulation platform was built (Figure 5). Notably, the data transmission of the scheduler and the controller adopted the Send module, which could modify the scheduling waveform in real time at the trigger port according to the optimal control calculation process and the game iteration result during the, respectively, sampling moment. In the overall time period, the historical Trigger input of the corresponding Send module would display a trapezoidal or triangular waveform.

Given the actual control process of a certain type of aeroengine at altitude $10 \mathrm{~km}$ and Mach number 1.5, the parameter matrix of plant is defined as 



FIgURE 5: True time simulation platform of HOSMC with noncooperative game scheduling.

$$
\begin{aligned}
A & =\left[\begin{array}{ccc}
0.879 & 0.111 & -0.010 \\
-0.007 & 0.952 & -0.006 \\
0.463 & -0.170 & 0.686
\end{array}\right], \\
B & =\left[\begin{array}{l}
0.019 \\
0.017 \\
0.143
\end{array}\right], \\
C^{\prime \prime} & =\left[\begin{array}{lll}
1 & 0 & 0 \\
0 & 1 & 0 \\
0 & 0 & 1
\end{array}\right] .
\end{aligned}
$$

First, the scheduling matrices of state variables and control variables are initialized, respectively, as

$$
\begin{aligned}
& \Psi(0)=\left[\begin{array}{lll}
1 & 0 & 0 \\
0 & 1 & 0 \\
0 & 0 & 1
\end{array}\right], \\
& M(0)=1 .
\end{aligned}
$$

After Jordan standardization, the transform item can be yielded as $\gamma=[9454-1116071]$, and the relevant parameter matrix can be converted into:

$$
\begin{aligned}
& \Upsilon=\left[\begin{array}{ccc}
9454 & -11160 & 71 \\
8421 & -9587 & 21 \\
7479 & -8195 & -12
\end{array}\right], \\
& \widetilde{A}=\left[\begin{array}{ccc}
0 & 1 & 0 \\
0 & 0 & 1 \\
0.578 & -2.097 & 2.517
\end{array}\right], \\
& \widetilde{B}=\left[\begin{array}{l}
0 \\
0 \\
1
\end{array}\right] .
\end{aligned}
$$

Based on the mentioned results, set the initial network patency degree $\widehat{\Psi}_{i i}(0)=0$, contribution degree $\kappa_{i}^{\text {th }}=4.35$, noise interference degree $\theta^{2}=0.3$, contribution degree regulatory factor $\varepsilon=1.5$, and cost factor $\sigma=1.1$. And, the number of iterations is set to 100 .

Through the horizontal comparison of each iteration calculation of (18), the minimum scheduling value is removed, and the total restriction of state is ensured to be 1 . In addition, the overall waveform of the scheduling signal corresponding to the three states is obtained (Figure 6).

According to Figure 6, the noncooperative game scheduling method proposed in the present study can maintain the transmission of the state signal at the level of $2 /$ 3 of the conventional NCS, effectively lifting the burden of the bus network.

The Figures 7(a) and 7(b) illustrate the game process at $0.5 \mathrm{~s}$ and $1 \mathrm{~s}$, respectively. It illustrates the rapid convergence and stability of the proposed algorithm, thereby helping reduce the holding time of the scheduler. 


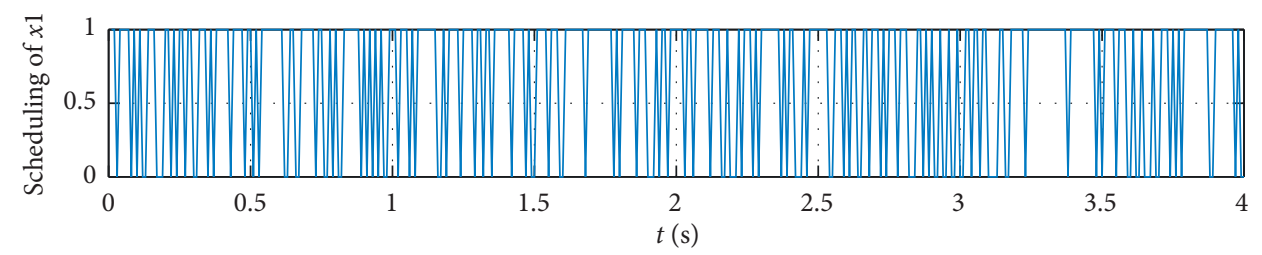

(a)

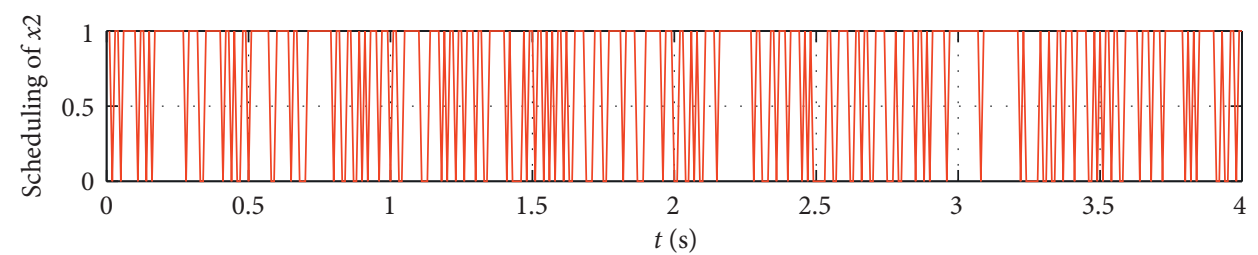

(b)

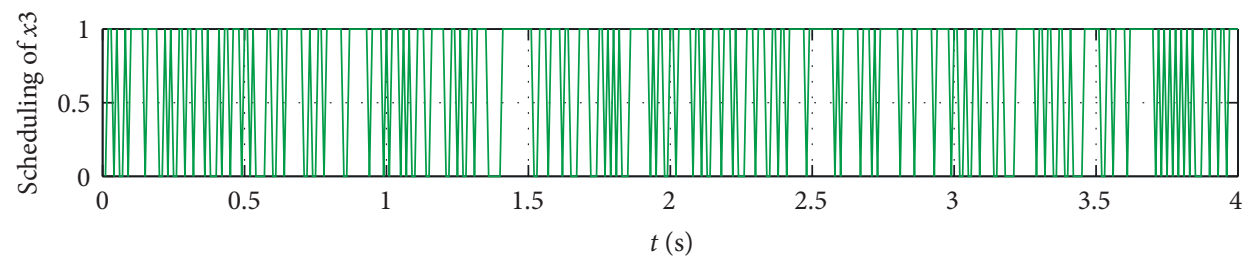

(c)

FIGURE 6: The corresponding transmission waveform of state noncooperative game scheduling.

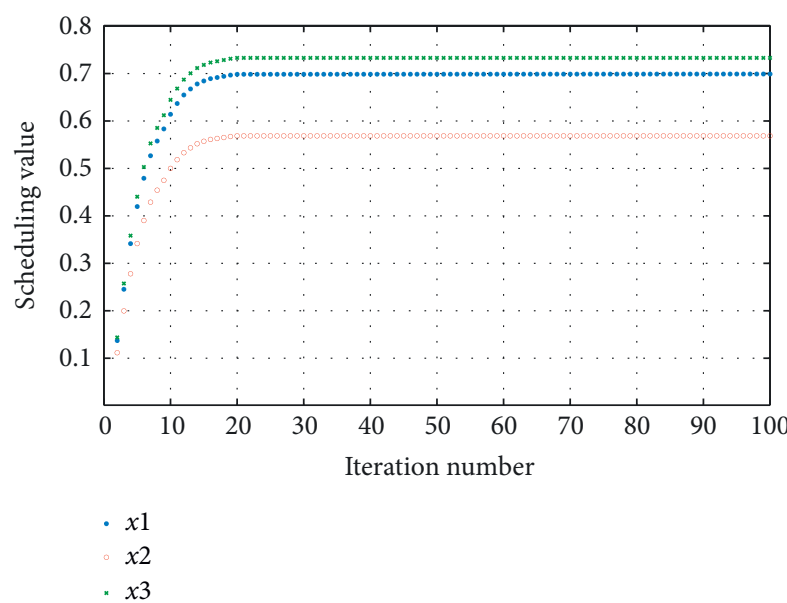

(a)

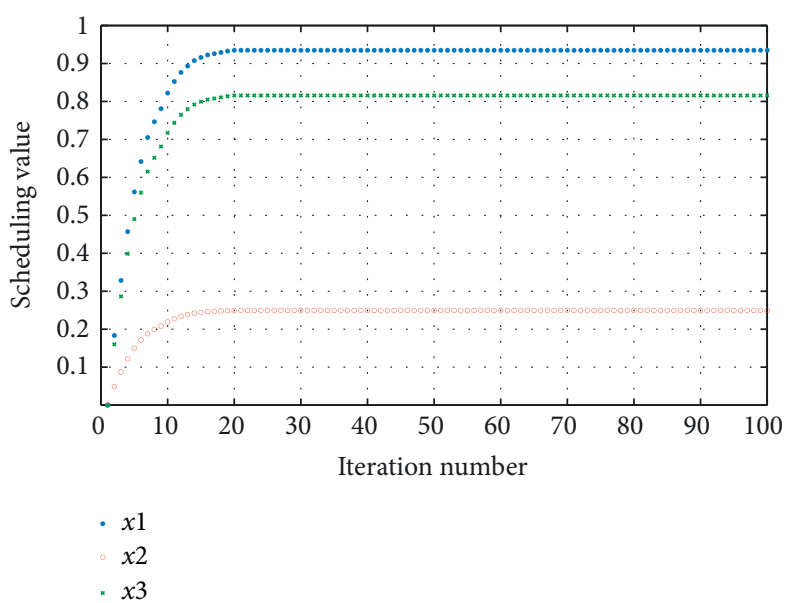

(b)

FIGURE 7: Iteration calculation results of state noncooperative game. (a) $0.5 \mathrm{~s}$; (b) $1 \mathrm{~s}$.

After verifying the effectiveness of the noncooperative game strategy in the limited network service, the control results are given to verify the rationality of the HOSMC algorithm.
By taking the appropriate objective function parameter matrix $P$ and $Q$, based on Theorem 3, the positive definite item of Riccati equation and the first-order linear sliding mode parameter are yielded as 


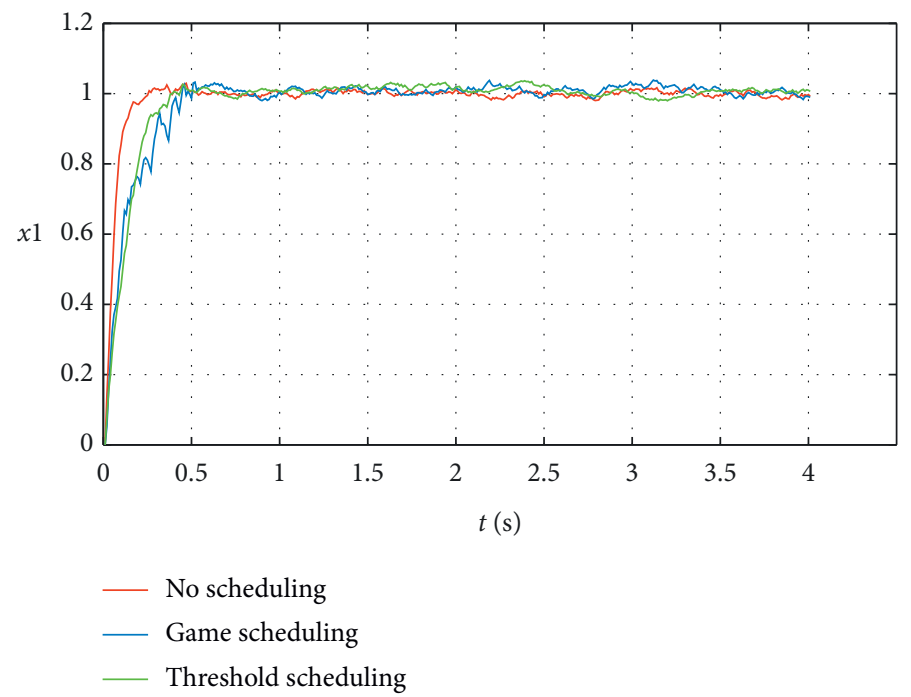

(a)

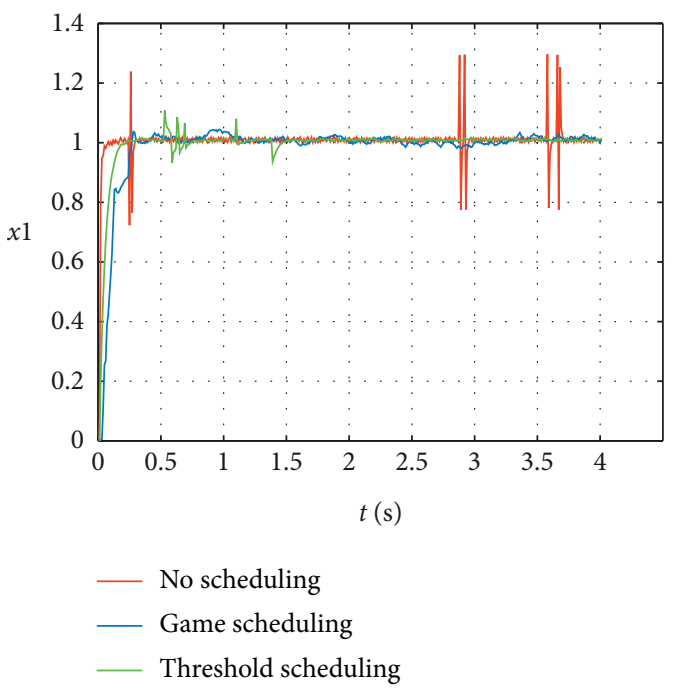

(b)

FiguRE 8: Step response under different scheduling strategies with interference. (a) $10 \%$ band interference; (b) $60 \%$ band interference.

$$
\begin{aligned}
& P=\left[\begin{array}{ll}
0.749 & 0.327 \\
0.327 & 0.749
\end{array}\right], \\
& Q=0.535, \\
& R=\left[\begin{array}{ll}
0.749 & 0.327 \\
0.327 & 1.444
\end{array}\right], \\
& C=\left[\begin{array}{ll}
0 & 0.165
\end{array}\right] .
\end{aligned}
$$

Through the Interference module to create various degrees of noise interference and bandwidth occupation atmosphere, under the action of the optimal cooperative controller, the step response of system is obtained. To reflect the advantages of the method proposed here, the adaptive SMC based on static threshold scheduling acts as a comparison method. The comparison result is illustrated in Figure 8.

According to Figure 8(a), when the bus transmission environment is relatively smooth, the settling time and steady-state error of the HOSMC algorithm under dynamic game scheduling in the present study were slightly different from the threshold scheduling method, whereas there were some fluctuations in the arrival stage. The reason was that the sending of a sensor state variable was always restricted here, while other types of network QoS service strategies were either in the "nonintervention" state or in the "full valve" state, so the method proposed here was relatively disadvantaged for system QoP. Combined with the conventional control results without scheduling, the collaborative control based on the noncooperative game scheduling is suggested to be at the expense of a little control quality in exchange for lifting the burden imposed on the network.

According to Figure 8(b), with the increase of noise interference and the decrease of available bandwidth, the effectiveness of this method gradually turned out to be prominent. Though the dynamic performance of system displayed a certain degradation, it exhibited better network congestion fault tolerance and anti-interference ability in terms of state maintenance and overall control quality.

As revealed from the analysis above, as opposed to adaptive SMC with static threshold scheduling, HOSMC with dynamic noncooperative game scheduling proposed in the present study could enhance the initiative and intelligence to deal with network adverse conditions. In addition, it was ensured that the network QoS environment was further dynamically optimized under the premise that QoP is not significantly weakened.

For control signal transmission, the given quadratic optimal control design has related adjustment items. It can be determined whether the data packet is restricted. Accordingly, to visually present the effectiveness of this part of the theory, a certain initial disturbance is set. Subsequently, the state convergence of the overall NCS is yielded, and the actual control input and the optimize control scheduling values are, respectively, presented in Figures 9 and 10.

According to the mentioned 2 figures, though in the limited network service under the effect of state noncooperative game scheduling strategy, the optimal high-order sliding mode cooperative controller proposed in the present study could still ensure that the system convergence characteristics is identical with expectation. In addition, the actual input after switching compensation did not show significant deviations and fluctuations, further demonstrating the rationality of the designed collaborative control theory. It is noteworthy that according to the control scheduling curve, the data packet sending situation in the second half of the feedback link could be regulated timely, which could additionally reduce the burden imposed on the bus and optimize the network QoS service environment. 


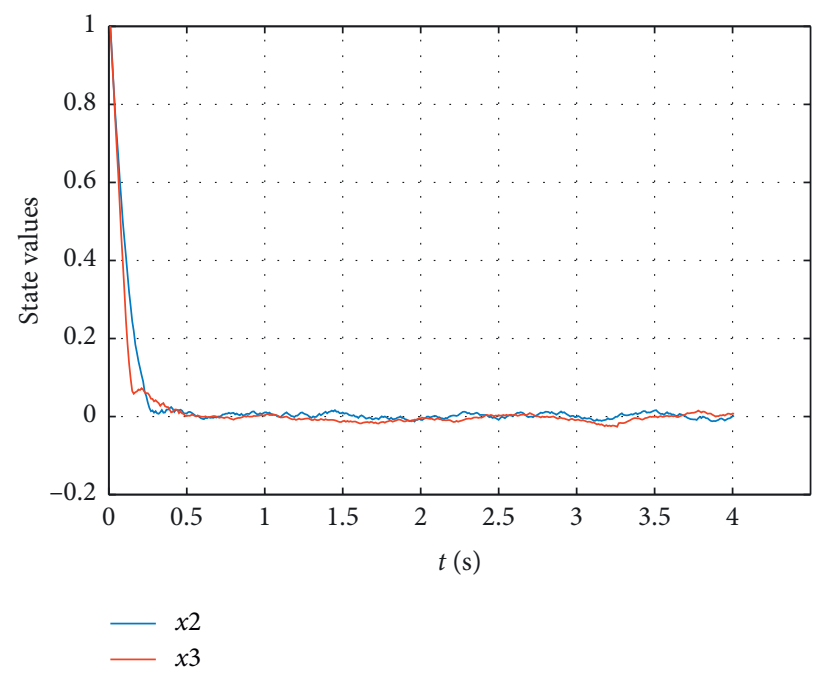

FIGURE 9: Initial disturbance response of system.

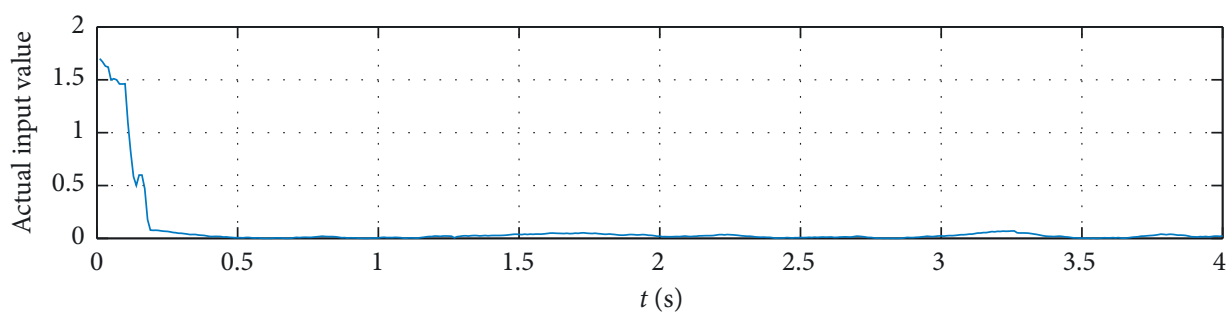

(a)

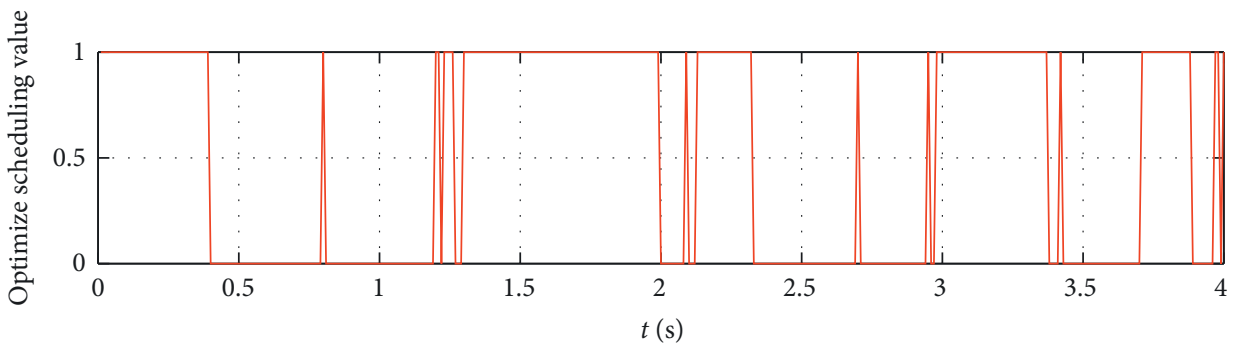

(b)

FIgURE 10: The control input and scheduling value corresponding to initial disturbance response.

Analyzing from the perspective of QoP, for the identical research object, using sine waves as the reference input signal, the HOSMC with noncooperative game scheduling proposed in the present study could be compared with the adaptive SMC with threshold scheduling (Figure 11).

To explore the reason for this phenomenon, since the quadratic optimal sliding surface of Theorem 4 cannot be expressed explicitly, here is a direct comparison of the control values of the two controllers, as presented in Figure 12.
Thus, the control value of HOSMC was generally not smooth enough by itself because the dynamics of the game scheduler determines the uncertainty of the equivalent control form, thereby causing the constant deviation and fluctuations of related calculation results. Combining Figures 11 and 12, the effectiveness of the proposed HOSMC with state dynamic noncooperative game scheduling proposed in the present study is again proved. 


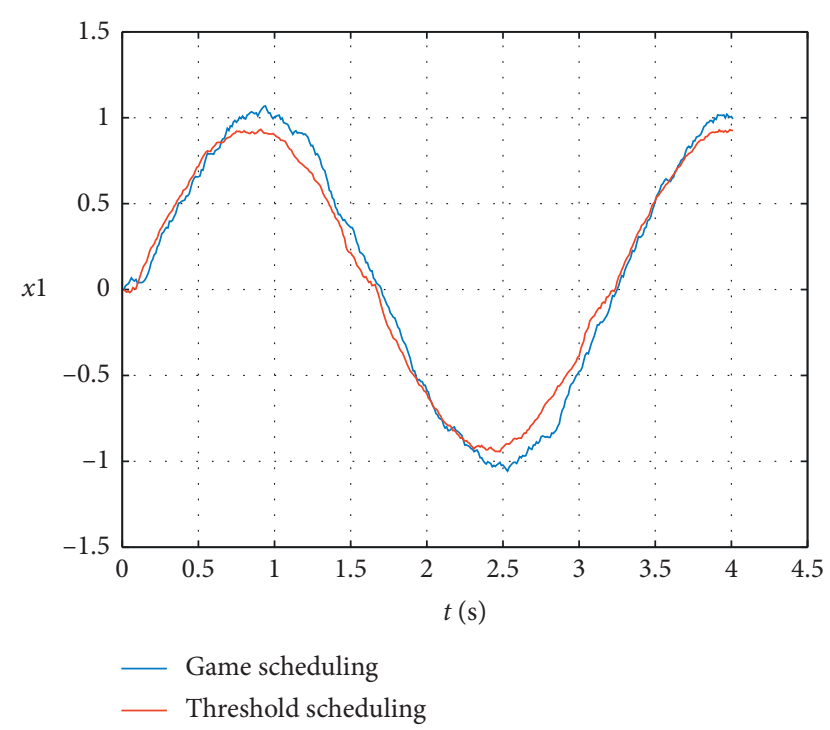

FIGURE 11: Comparison of tracking control between noncooperative game HOSMC and threshold adaptive SMC.

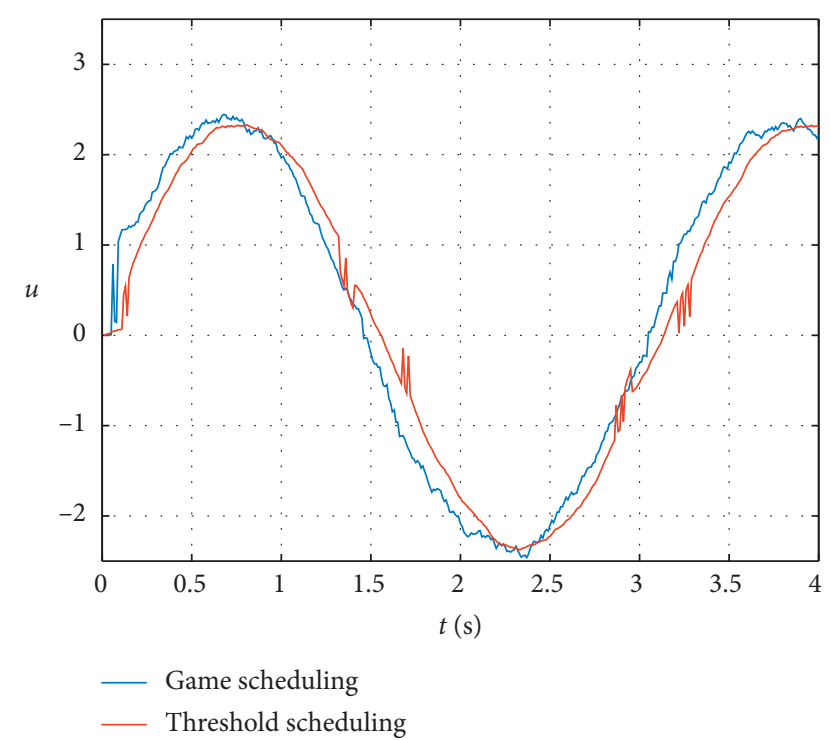

Figure 12: Comparison of the control values of the two control methods.

\section{Conclusions}

In the present study, based on the Nash game theory, the network dynamic scheduling strategy for states and cooperative control theory of the NCS were studied. The balance of network QoS and QoP control were achieved, and the overall control system's ability to actively respond to poor service environments (e.g., network congestion and noise interference) was enhanced.

Firstly, using the transformation of the state space equation form of the research object to build the necessary conditions for the establishment of the noncooperative game model, the three elements of the game were established, and the existence and uniqueness of the Nash equilibrium solution were proved. Through the derivation of the utility function and the matching iterative equation, the dynamic noncooperative game scheduling transmission law for state was finished.

Secondly, the HOSMC was selected as the cooperative control method, and its nonlinear degree, i.e., the sliding mode order, should refer to the number of restricted states. Subsequently, considering the effect of the game strategy and the synchronous control scheduling, the equivalent control adjustment item and the quadratic optimal time-varying sliding mode surface were designed. After embedding the switching item into $\mathrm{ZOH}$, the research on HOSMC with state game scheduling was completed.

Lastly, the corresponding TrueTime simulation platform was built, and the results of the examples verified the dynamic, intelligence, and superiority of the proposed method in the limited bandwidth and strong interference network atmosphere, as well as the effectiveness of QoP quality under ideal transmission conditions. It was therefore verified that the research in the present study has fulfilled the aim of overall improvement of network services and control performance of NCSs.

Future work will focus on two aspects. Theoretically, the influence of network induced problem on system stability will be considered, and other scheduling methods such as event-triggered scheduling will be adopted to optimize the system performance. In the aspect of experiment, for a practical control system, it is not enough to rely solely on computer simulation. Therefore, we will focus on the semiphysical platform of NCS based on CAN bus network to realize the hardware-in-the-loop verification of the control theory.

\section{Data Availability}

The data used to support the findings of this study are already included within the article.

\section{Conflicts of Interest}

The authors declare no conflicts of interest.

\section{Acknowledgments}

This work was supported in part by the National Natural Science Foundation of China under grants 51606219 and 51476187.

\section{References}

[1] X. Zhang, Q. Han, X. Ge et al., "Networked control systems: a survey of trends and techniques," IEEE/CAA Journal of Automatica Sinica.vol. 7, no. 1, pp. 1-17, 2020.

[2] R. S. Raji, "Smart networks for control," IEEE Spectrum, vol. 31, no. 6, pp. 49-55, 1994.

[3] L. Chen, J. Zhang, and S. Wang, "Scheduling and control codesign for delay compensation in the networked control system," Asian Journal of Control, vol. 8, no. 2, pp. 124-134, 2006.

[4] B. Zhou, S. Xie, F. Wang, and J. Hui, "Multi-step predictive compensated intelligent control for aero-engine wireless 
networked system with random scheduling," Journal of the Franklin Institute, vol. 357, no. 10, pp. 6154-6174, 2020.

[5] P. Wang, Z. Liu, Q. Liu, and H. Chen, "An MPC-based manoeuvre stability controller for full drive-by-wire vehicles," Control Theory and Technology, vol. 17, no. 4, pp. 357-366, 2019.

[6] J. Wang, M. Zhou, and C. Liu, "Stochastic stability of Markovian jump linear systems over networks with random quantization density and time delay," Physica A: Statistical Mechanics and Its Applications, vol. 509, pp. 1128-1139, 2018.

[7] F. Cai, J. Huang, W. Wang, J. Huang, Q. Lin, and Y. Li, " $H \infty$ filtering of networked switched systems with multiple packet dropouts via switched Lyapunov function approach," IET Control Theory \& Applications, vol. 14, no. 9, pp. 1220-1227, 2020.

[8] S. Xiong, M. Chen, and Q. Wu, "Predictive control for networked switch flight system with packet dropout," Applied Mathematics and Computation, vol. 354, pp. 444-459, 2019.

[9] Z. Wang and S. Fukushima, "Control strategy for networked control systems with time delay and packet dropout using linear matrix inequalities," EURASIP Journal on Wireless Communications and Networking, vol. 2020, no. 3, pp. 1-5, 2020.

[10] Q. Geng, F. Liu, and H. Yang, "Self-triggered sampling control for networked control systems with delays and packets dropout," International Journal of Systems Science, vol. 49, no. 8, pp. 1703-1714, 2018.

[11] B. Lian, Q. Zhang, and J. Li, "Integrated sliding mode control and neural networks based packet disordering prediction for nonlinear networked control systems," IEEE Transactions on Neural Networks and Learning Systems, vol. 30, no. 8, pp. 2324-2335, 2019.

[12] B. Lian, Q. Zhang, and J. Li, "Sliding mode control and sampling rate strategy for Networked control systems with packet disordering via Markov chain prediction," ISA Transactions, vol. 83, pp. 1-12, 2018.

[13] D. Zhang, Q.-L. Han, and X.-M. Zhang, "Network-based modeling and proportional-integral control for direct-drivewheel systems in wireless network environments," IEEE Transactions on Cybernetics, vol. 50, no. 6, pp. 2462-2474, 2020.

[14] F. Lian, J. Moyne, and D. Tilbury, "Network design consideration for distributed control systems," IEEE Transactions on Control Systems Technology, vol. 10, no. 2, pp. 297-307, 2002.

[15] W. Chen, J. Yao, and L. Qiu, "Networked stabilization of multi-input systems over shared channels with scheduling/ control co-design," Automatica, vol. 99, pp. 188-194, 2019.

[16] Y. Yuan, P. Zhang, Z. Wang, and Y. Chen, "Noncooperative event-triggered control strategy design with round-robin protocol: applications to load frequency control of circuit systems," IEEE Transactions on Industrial Electronics, vol. 67, no. 3, pp. 2155-2166, 2020.

[17] Q. Ma, C. Zhou, and X. Zhu, "Event-trigger-based hybrid scheduling and feedback control for networked control systems," IMA Journal of Mathematical Control and Information, vol. 34, no. 2, pp. 683-696, 2017.

[18] J. Hu, H. Zhang, X. Yu, H. Liu, and D. Chen, "Design of sliding-mode-based control for nonlinear systems with mixed-delays and packet losses under uncertain missing probability," IEEE Transactions on Systems, Man, and Cybernetics: Systems, no. 99, pp. 1-12, 2019.

[19] J. Song, Z. Wang, and Y. Niu, "Static output-feedback sliding mode control under round-robin protocol," International
Journal of Robust and Nonlinear Control, vol. 28, no. 18, pp. 5841-5857, 2018.

[20] J. Song, Z. Wang, and Y. Niu, "On $\$ H_{-}$linfty\$ sliding mode control under stochastic communication protocol," IEEE Transactions on Automatic Control, vol. 64, no. 5, pp. 21742181, 2019.

[21] B. Zhang, Q. Han, X. Zhang, and X. Yu, "Sliding mode control with mixed current and delayed states for offshore steel jacket platforms," IEEE Transactions on Control Systems Technology, vol. 22, no. 5, pp. 1769-1783, 2014.

[22] X. Shao and H. Wang, "Sliding mode based trajectory linearization control for hypersonic reentry vehicle via extended disturbance observer," ISA Transactions, vol. 53, no. 6, pp. 1771-1786, 2014.

[23] A. Chalanga and F. Plestan, "High-order sliding-mode control with predefined convergence time for electropneumatic actuator," IEEE Transactions on Control Systems Technology, vol. 29, no. 2, pp. 910-917, 2021.

[24] J. Neumann, "Zur theorie der Gesellschaftsspiele," Mathematische Annalen, vol. 100, no. 1, pp. 295-320, 1928.

[25] F. Takako, Noncooperative Game Theory, Springer, Tokyo, Japan, 2015.

[26] Q. La, Y. Chew, and B. Soong, Potential Game Theory, Springer, Cham, Switzerland, 2016.

[27] Y. Tipsuwan, S. Kamonsantiroj, J. Srisabye, and P. Chongstitvattana, "An auction-based dynamic bandwidth allocation with sensitivity in a wireless networked control system," Computers \& Industrial Engineering, vol. 57, no. 1, pp. 114-124, 2008.

[28] X. Yan, Y. Sun, and B. Mei, "Dynamic bandwidth scheduling of software defined networked collaborative control system," IEEE Access, vol. 8, pp. 75968-75975, 2020.

[29] L. Zhang, S. Zhang, L. Wu, Y. Liu, and C. Zhao, "A nash game algorithm for distributive power control with faster convergence in cognitive radio," in Proceedings of the 2009 9th International Symposium on Communications and Information Technology, pp. 93-96, Incheon, South Korea, September 2009.

[30] O. Kolditz, Computational Methods in Environmental Fluid Mechanics, Springer, Berlin, Heidelberg, 2002.

[31] N. K. Sharma and S. Janardhanan, "Discrete higher order sliding mode: concept to validation," IET Control Theory \& Applications, vol. 11, no. 8, pp. 1098-1103, 2017.

[32] X. Yu, B. Wang, Z. Galias, and G. Chen, "Discretization effect on equivalent control-based multi-input sliding-mode control systems," IEEE Transactions on Automatic Control, vol. 53, no. 6, pp. 1563-1569, 2008. 